\title{
Intravenous diuretic day-care treatment for patients with heart failure
}

\author{
P Banerjee, G Tanner and L Williams
}

\begin{abstract}
Fluid overload is a common manifestation of decompensated chronic heart failure. This paper reports on a pilot study that investigated whether intravenous (iv) furosemide administered on a cardiology day ward for three successive days was effective in improving the symptoms of patients with fluid overload and chronic heart failure. The results showed that $94.1 \%$ of patients reported an improvement in their breathlessness, with a marked weight loss in $88.2 \%$ of patients. There were no marked changes in blood pressure or renal function. Hospital admission was avoided in $94.1 \%$ of cases. The study concluded that iv diuretic treatment given in a hospital day-care setting is safe and effective, and that it reduces the need for hospital admissions. As a consequence, this reduces the associated financial costs of hospitalisation.
\end{abstract}

KEY WORDS: day care, heart failure, intravenous diuretics

\section{Introduction}

Fluid overload is a common manifestation of decompensated chronic heart failure. To treat this, increasing doses of oral loop diuretics, such as furosemide and bumetanide, are frequently used as first-line treatment ${ }^{1,2}$ and the addition of a second, usually thiazide-type, diuretic, such as bendroflumethiazide ${ }^{3}$ or metolazone, ${ }^{4}$ is used as second-line treatment for incremental diuresis. Hospital admission for intravenous (iv) diuretics is often required in cases where the first two approaches are ineffective., ${ }^{5,6}$ A day-care approach for iv furosemide treatment was devised to avoid the need for hospital admission by administering the drug on the cardiology day-care ward for three successive days. This was a prospective pilot project available only to patients with heart failure who were being managed by the cardiology team in the first instance. If successful, the aim was to extend this service to all suitable patients with heart failure who were managed by other hospital teams, such as elderly medicine, acute medicine, and so on.

\section{Methods}

\section{Inclusion and exclusion criteria}

The project was conducted in a large teaching hospital (University Hospitals Coventry \& Warwickshire) in Coventry,

P Banerjee, consultant cardiologist and lead, heart failure unit; G Tanner, heart failure specialist nurse; L Williams, lead nurse, heart failure unit

University Hospitals Coventry and Warwickshire
UK. As the role of iv furosemide is well established in heart failure, approval from the local ethics committee was not deemed necessary. The use of day-care iv diuretic treatment (DCIDT) was confined to patients with either fluid overload and left ventricular systolic dysfunction (LVSD), or fluid overload, heart failure and normal ejection fraction (HFNEF). Those patients with pure right heart failure were excluded. It was important to select patients for DCIDT carefully by ensuring that those who were selected were not too ill and/or fluid overloaded to benefit from only three days of iv diuretics. It was emphasised that if the doctor assessing the patient felt that more than three days of iv diuretics would be required to achieve adequate fluid offloading, it would be better to admit the patient to hospital than to attempt day-care therapy.

The main indication of DCIDT treatment was the need to use iv diuretics to treat fluid overload caused by heart failure in patients who were safe to receive this form of treatment. This included those patients who were felt to have failed a trial of enhanced oral diuresis or were deemed more suitable for direct iv therapy rather than attempting enhanced oral diuresis. Patients already admitted to the Heart Failure Unit of the hospital could also be considered for DCIDT, with the plan that they would be discharged early from the hospital after initial clinical stabilisation with inpatient iv diuretics but brought back as day-care patients to continue with iv diuresis. Definite exclusions for DCIDT comprised those patients with acute pulmonary oedema, cardiogenic shock, a habitual systolic blood pressure (BP) of $<90 \mathrm{mmHg}$, acute coronary syndrome with fluid overload, complex heart failures with several comorbidities needing more intense monitoring in hospital, renal failure (creatinine $>200 \mathrm{mmol} / \mathrm{l}$ ), valvular heart disease with heart failure who needed working up, those patients likely to need more than three days of iv diuretics to benefit and those who were too old or frail to make daily visits to the hospital.

\section{Form and referral}

A paper form was created to enable referrals for DCIDT to be made (Fig 1). This form was also used for day-care iv iron treatment in patients with heart failure. Referring cardiology doctors filled the form out in the cardiology outpatient clinic and rang the cardiology day ward to arrange dates for the treatment with the nurse in charge, who then confirmed the dates after checking with the DCIDT scheduler. As the treatment involved three successive days and there was no available service on a Saturday or Sunday, patients were booked in to begin treatment on 


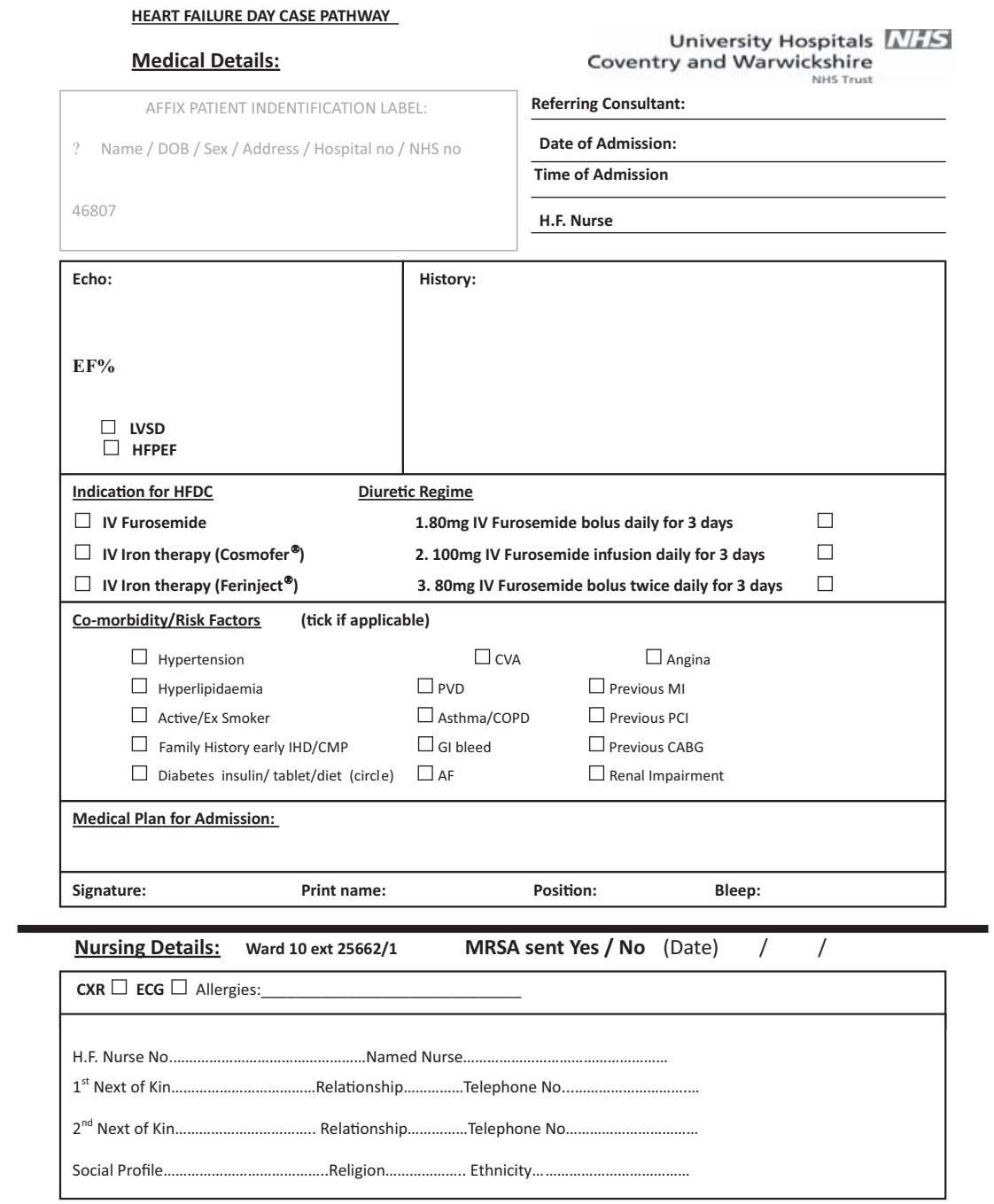

Fig 1. Combined form for DCIDT and iv iron.

Mondays, Tuesdays and Wednesdays only. Once confirmed, the clinic nurse informed the patient about the dates and/or times for DCIDT and checked that appropriate travel arrangements were in place.

\section{The DCIDT process}

The iv diuretic treatment was offered either as a daily double bolus or as single treatment in the form of a bolus and/or infusion of iv furosemide for three consecutive days. The double bolus consisted of twice-daily $80 \mathrm{mg}$ iv bolus doses for three consecutive days, ideally suited for patients who were markedly fluid overloaded. The alternative, for those patients who were less overloaded, was the single treatment of a daily $80 \mathrm{mg}$ iv bolus or a $100 \mathrm{mg}$ iv infusion with a further later oral furosemide dose and/or a small dose of metolazone to be taken at home for three consecutive days. The indication and the mode of iv diuretic administration were recorded on the DCIDT form by the doctor requesting the treatment. Choosing the single treatment option was encouraged because it enabled better use of time, as up to a maximum of two patients could be booked in per day (at 9am and $1 \mathrm{pm})$. The anticipated stay for each patient was 3.5 hours. For the double-bolus treatment, only one patient could be booked in per day.

On each visit to the day-care ward, the patient's blood pressure, pulse, weight, New York Heart Association (NYHA) class and their own assessment of daily urine output were checked and recorded on the day-care form by the junior doctor seeing the patient. The care of the patient during their brief visit was shared by the nurses on the day ward and a junior doctor. Bloods were taken and sent off for a full blood count (FBC) and urea and electrolytes (U\&Es) test on each day of treatment, and a blood form was given to the patient to have their bloods checked on the fourth day. These fourth-day bloods results were checked by the patient's general practitioner (GP) and the community heart failure specialist nurse looking after the patient. On discharge from this treatment, a discharge summary was sent to the patient's GP. The oral diuretic regimen to continue was decided by the cardiology firm looking after the patient.

\section{Results}

In total, 17 patients underwent DCIDT over 1 year at the University Hospitals Coventry \& Warwickshire. The patients were receiving standard medical therapy for heart failure. Their mean age was $70 \pm 6$ (range 48-93) years, with 12 males $(70.6 \%)$ and five females $(29.4 \%)$. Eleven patients $(64.7 \%)$ were referred from the community heart failure service, four $(23.5 \%)$ from the diagnostic heart failure clinic of the hospital (having been newly diagnosed with heart failure) and two $(11.8 \%)$ from palliative care with known end-stage heart failure. The comorbidities in these patients are outlined in Fig 2. The cause of decompensation was unknown in 13 patients $(76.5 \%)$, non-concordance to medications in two $(11.8 \%)$, addition of new drugs in one (5.9\%) and infection in another (5.9\%). Out of the 17 patients, only one $(5.9 \%)$ had heart failure with normal ejection fraction; the other 16 patients $(94.1 \%)$ had left ventricular systolic dysfunction. The iv furosemide was administered as a single $100 \mathrm{mg}$ infusion in 12 patients (70.6\%), a single $80 \mathrm{mg}$ bolus in two patients (11.8\%) and as two separate $80 \mathrm{mg}$ boluses 4 hours apart in three patients (17.6\%).

Unfortunately, no record was kept of the number of patients screened for this type of treatment. The number screened for DCIDT was estimated to be approximately three times the number who subsequently were suitable for the treatment. It is 
important to note that the some of the screened patients were unsuitable because daily transport to the hospital was difficult to arrange.

In total, 16 patients $(94.1 \%)$ reported feeling better with improved breathing. The NYHA class dropped by half a class in 12 of these patients $(70.5 \%)$ and by one full class in four patients $(23.5 \%)$; in one patient $(6 \%)$, who had HFNEF, there was no change in NYHA class. As shown in Fig 3, there was weight loss of $0.5-6.0 \mathrm{~kg}$ in 15 patients $(88.2 \%)$, but weight gain in two patients $(11.8 \%)$. There were no marked changes in BP or electrolytes and renal function, as shown in Fig 4. At the end of the treatment, 16 patients $(94.1 \%)$ were discharged back to the heart failure nursing service for follow-up. However, one patient (who had HFNEF) showed no signs of improvement and had to be admitted to hospital. Therefore, hospital admission was prevented in $94.1 \%$ of cases, resulting in an estimated saving of approximately $\mathfrak{E} 35,200$ ( $€ 40,000$ ).

Out of the 17 patients in the trial, 14 were not readmitted within 28 days. However, there was one heart-failure-related readmission, which occurred within 14 days as a result of hypokalaemia. Two other patients were readmitted for non-heart-failure reasons within 28 days; one with bronchopneumonia and the other with cellulitis of the legs.

The only patient who needed admission had HFNEF with a background of hypertension, type 2 diabetes mellitus and chronic renal failure. His treatment included furosemide $40 \mathrm{mg}$ daily, alendronate $70 \mathrm{mg}$ weekly, digoxin $62.5 \mu \mathrm{g}$ daily, rabeprazole $10 \mathrm{mg}$ daily, hydralazine $25 \mathrm{mg}$ three times daily and isosorbide mononitrate slow-release (ISMN SR) $30 \mathrm{mg}$ daily.

\section{Conclusion}

Decompensated heart failure with fluid overload remains an important cause of frequent hospital admissions for iv diuretic therapy. A previous retrospective study found outpatient iv diuretic therapy to be safe and effective. ${ }^{7}$ Our pilot study examined an innovative way of providing iv diuretics (DCIDT) in an effective manner within the hospital setting without having to admit the patient. The three-day regimen was safe in that it did not cause any significant drop in blood pressure or derangement of renal function or electrolytes. There was improvement of breathlessness in $94.1 \%$ of patients and weight loss occurred in $88.2 \%$. Hospital admission was prevented in $94.1 \%$ of cases. The financial gain as a result of preventing hospital admission was substantial. disease.

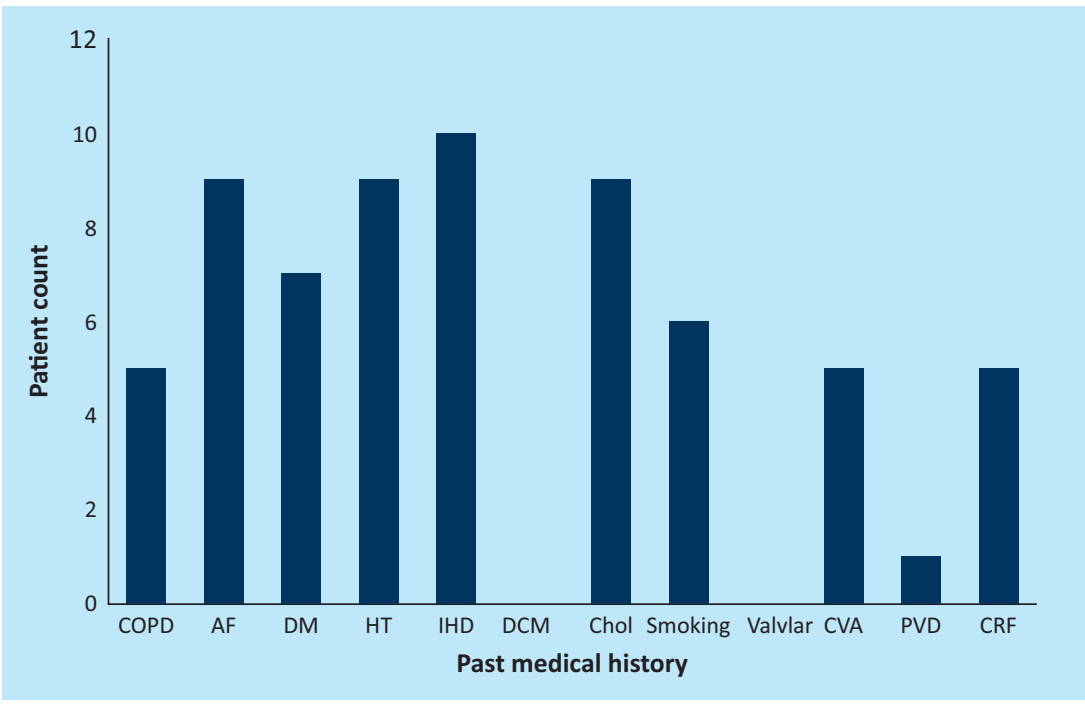

Fig 2. Co morbidities in patients in the trial $(n=17)$. $A F=$ atrial fibrillation; $\mathrm{Chol}=$ cholesterol; $\mathrm{COPD}=$ chronic obstructive pulmonary disease; $\mathrm{CRF}=$ chronic renal failure; mellitus; HT = hypertension; IHD = ischaemic heart disease; PVD = peripheral vascular

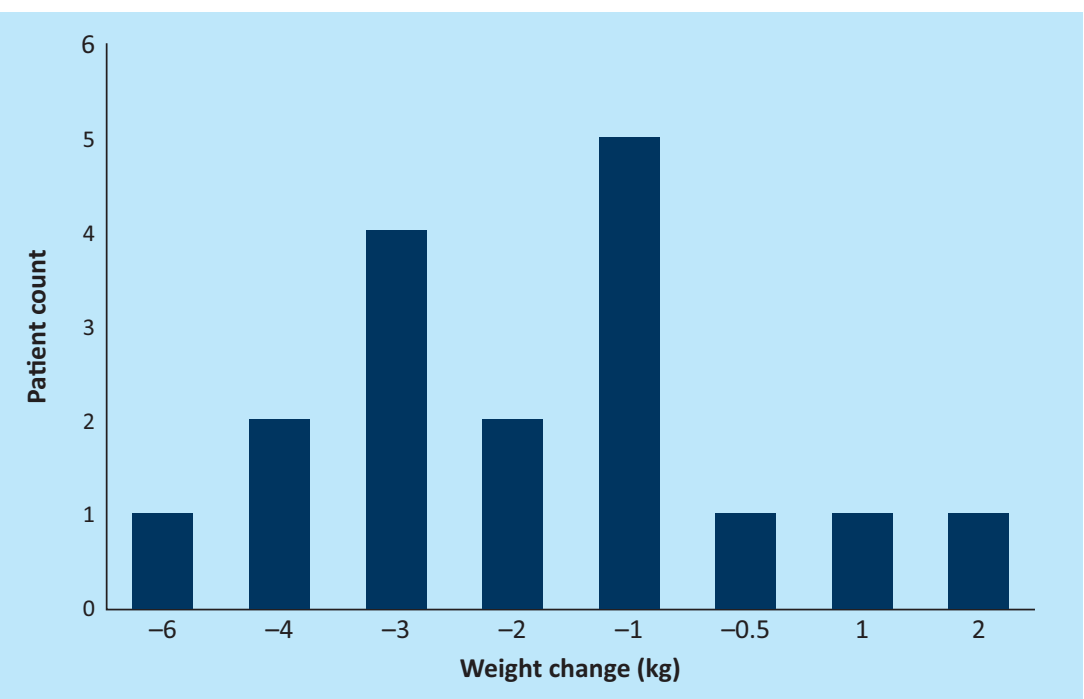

Fig 3. Weight change on day 3 of the treatment.

Although new services aimed at developing iv diuretics in the community are starting to be developed, our pilot study was successful in both preventing hospital admission and treating patients with fluid overload effectively in the day-care setting. Therefore, DCIDT might be a service that can be adopted for patients attending hospital clinics or acute medical units. Such a service would also fit well alongside a community iv diuretic service and form part of a spectrum of iv diuretic services for patients with heart failure in the community, in hospital and in day-care units (ie as outpatients). The aim is to continue with this cost-effective service and expand it to include patients 


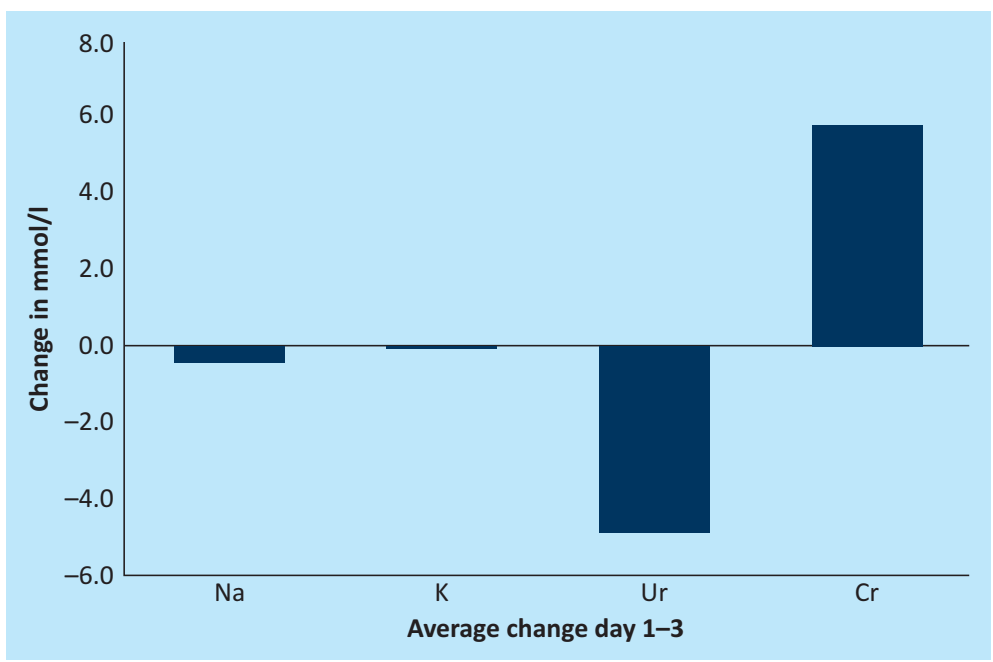

Fig 4. Change in electrolytes and renal function with IV diuretic.

attending other medical clinics or acute medicine, keeping the criteria for entry into the service strict. More experience will clarify whether patients with HFNEF should be excluded from the service.

\section{References}

1 Vasko MR, Brown-Cartwright D, Knochel JP et al. Furosemide absorption altered in decompensated congestive heart failure. Ann Intern Med 1985;102:314-8.
2 Johnson JA, Parker RB, Patterson JH. Heart failure. In: Dipiro JT, Talbert RL, Yee GC, Matzke GR, Wells BG et al. (eds) Pharmacotherapy: a pathophysiologic approach. New York: McGraw-Hill, 2002:185-218.

3 Dormans TP, Gerlag PG. Combination of high-dose furosemide and hydrochlorothiazide in the treatment of refractory congestive heart failure. Eur Heart $J$ 1996;17:1864-74.

4 Kiyingi A, Field MJ, Pawsey CC et al. Metolazone in treatment of severe refractory congestive cardiac failure. Lancet 1990;335:29-31.

5 Maisel A, Hollander JE, Guss D et al. Primary results of the Rapid Emergency Department Heart Failure Outpatient Trial (REDHOT). A multicenter study of B-type natriuretic peptide levels, emergency department decision-making, and outcomes in patients presenting with shortness of breath. $J$ Am Coll Cardiol 2004;44:1328-33.

6 Dormans TP, van Meyel JJ, Gerlag PG et al. Diuretic efficacy of high dose furosemide in severe heart failure: bolus injection versus continuous infusion. J Am Coll Cardiol 1996;28:376-82.

7 Ryder M, Murphy NF, McCaffrey D et al. Outpatient intravenous diuretic therapy: potential for marked reduction in hospitalisations for acute decompensated heart failure. Eur J Heart Fail 2008;10:267-72.
Address for correspondence: Dr P Banerjee,

University Hospitals Coventry and Warwickshire, Department of Cardiology, Clifford Bridge Road, Coventry, CV2 2DX.

Email: Prithwish.Banerjee@uhcw.nhs.uk

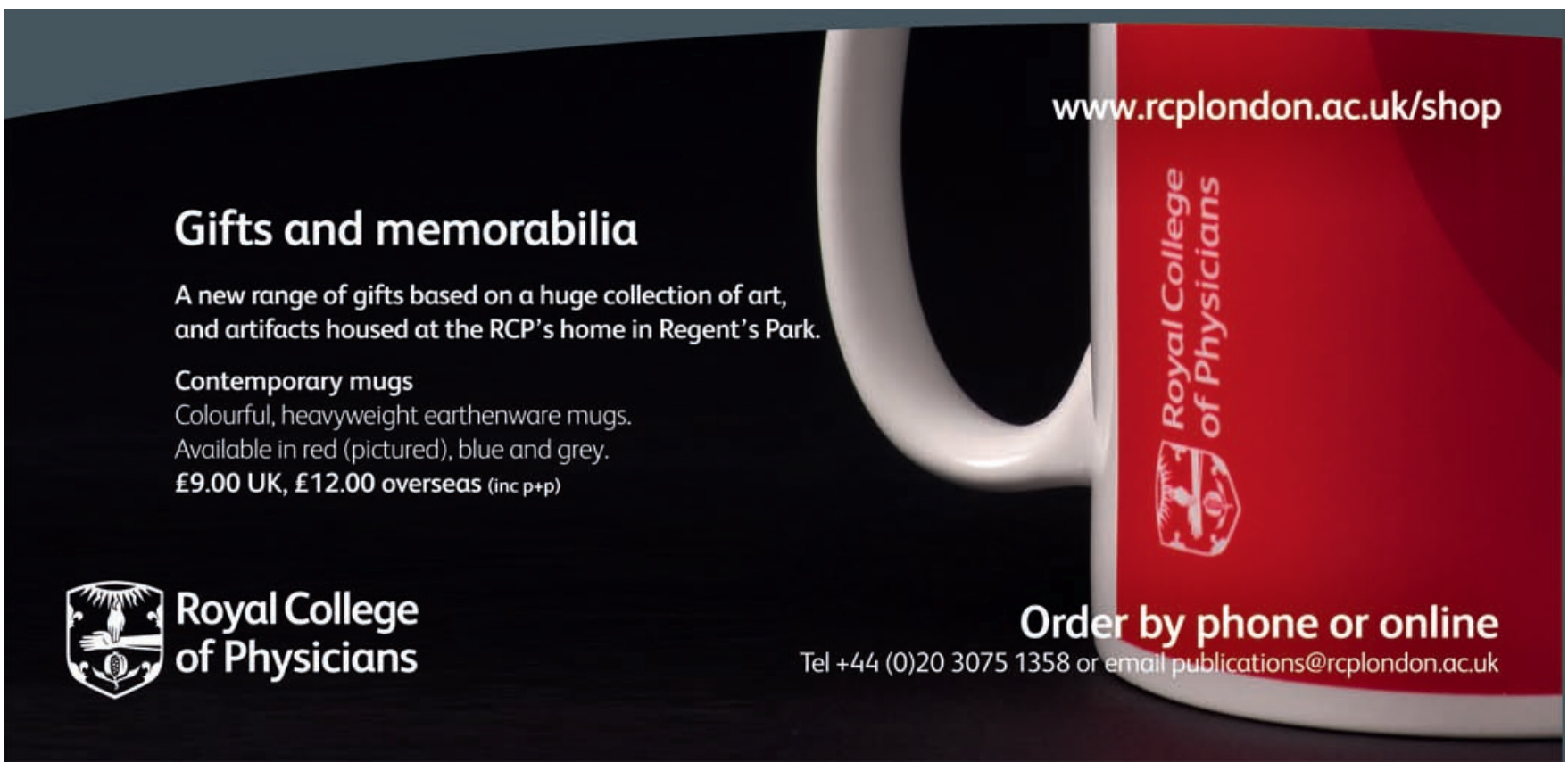

